Castro-Mejía et al.,

\title{
1 Gut microbiome and its cofactors are linked to 2 lipoprotein distribution profiles
}

3

4

5

$13{ }^{4}$ Institute of Experimental Endocrinology, Biomedical Research Center, University Science

14 Park for Biomedicine, Dúbravská cesta 9, 94505, Bratislava, Slovakia.

$15{ }^{5}$ Swammerdam Institute for Life Sciences, University of Amsterdam, Postbus 94215,

16 Amsterdam $1090 \mathrm{GE}$, The Netherlands

$17{ }^{6}$ Department of Plant and Environmental Sciences, University of Copenhagen, 1871

18 Frederiksberg C, Denmark

$19{ }^{7}$ School of Sport, Exercise and Rehabilitation Sciences, University of Birmingham, 20 Birmingham, B15 2TT, United Kingdom

* Corresponding author(s):

Josué L. Castro-Mejía (jcame@food.ku.dk) and Dennis S. Nielsen (ㅁ@@,food.ku.dk)

Rolighedsvej 26, Department of Food Science, University of Copenhagen, 1958

Frederiksberg C, Denmark

Josué L. Castro-Mejía ${ }^{1 *}$, Bekzod Khakimov ${ }^{1}$, Mads V. Lind ${ }^{2}$, Eva Garne ${ }^{1,3}$, Petronela

Paulová $^{1,4}$, Elnaz Tavakkoli ${ }^{5}$, Lars H. Hansen ${ }^{6}$, Age K. Smilde ${ }^{1,5}$, Lars Holm ${ }^{3,7}$, Søren B.

Engelsen ${ }^{1}$, Dennis S. Nielsen ${ }^{1 *}$

${ }^{1}$ Department of Food Science, University of Copenhagen, 1958 Frederiksberg C, Denmark

${ }^{2}$ Department of Nutrition, Exercise and Sports, University of Copenhagen, 1958

Frederiksberg C, Denmark

${ }^{3}$ Department of Biomedical Sciences, University of Copenhagen, 2200 Copenhagen N, 
Castro-Mejía et al.,

\section{Abstract}

Increasing evidence indicates that the gut microbiome (GM) plays an important role

34 in the etiology of dyslipidemia. To date, however, no in-depth characterization of the

35 associations between GM and its metabolic attributes with deep profiling of lipoproteins

36 distributions (LPD) among healthy individuals has been conducted. To determine associations and contributions of GM composition and its cofactors with distribution profiles of lipoprotein subfractions, we studied blood plasma LPD, fecal short-chain fatty acids (SCFA) and GM of 262 healthy Danish subjects aged 19-89 years.

Stratification of LPD segregated subjects into three clusters of profiles that reflected differences in the lipoprotein subclasses, corresponded well with limits of recommended levels of main lipoprotein fractions and were largely explained by host characteristics such as age and body mass index. Higher levels of HDL, particularly driven by large subfractions (HDL2a and HDL2b), were associated with a higher relative abundance of Ruminococcaceae and Christensenellaceae. Increasing levels of total cholesterol and LDL, which were primarily associated with large 1 and 2 subclasses, were positively associated with Lachnospiraceae and Coriobacteriaceae, and negatively with Bacteroidaceae and Bifidobacteriaceae. Metagenome sequencing showed a higher abundance of genes involved in the biosynthesis of multiple Bvitamins and SCFA metabolism among subjects with healthier LPD profiles. Metagenomic assembled genomes (MAGs) affiliated mainly to Eggerthellaceae and Clostridiales were

51 identified as the contributors of these genes and whose relative abundance correlated positively with larger subfractions of HDL. metabolic traits of the GM are associated with variations in LPD among healthy subjects. shade light on mechanisms of the GM - dyslipidemia axis.

58 Keywords: gut microbiome, SCFAs, lipoproteins distribution, HDL, ${ }^{1} \mathrm{H}$ NMR 
Castro-Mejía et al.,

\section{INTRODUCTION}

Cholesterol is essential for keeping cellular integrity and is an important precursor for steroid hormones and bile acids ${ }^{1}$. However, alterations of the cholesterol metabolism and consequent dyslipidemia have been associated with various diseases, including atherosclerosis and cardiovascular diseases (CVD) ${ }^{2}$, as well as breast cancer ${ }^{3}$.

Recent advances in metabolomics research have allowed large-scale and highthroughput profiling of lipoprotein distribution's (LPD) in human blood plasma based upon their composition and concentration ${ }^{4-6}$. It has been hypothesized that numerous medical conditions such as glucose intolerance, type-2 diabetes, myocardial infarction, ischemic stroke and intracerebral hemorrhage, might be associated with lower blood levels of larger HDL particles (e.g. HDL2a and HDL2b) and a higher content of triglycerides within the lipoproteins particles ${ }^{7,8}$.

During the last decade it has been shown that alterations in gut microbiome (GM) composition contribute to the development and progression of several metabolic and immunological complications ${ }^{9}$. Furthermore, a handful of recent studies on different cohorts have also demonstrated that the changes in intestinal microbiota are highly correlated to variations in levels of lipoproteins in blood ${ }^{10-12}$, as well as to promote atherosclerosis ${ }^{13}$, and regulate cholesterol homeostasis ${ }^{14}$.

The relationship between GM and LPD has only been scarcely investigated. Recently Vojinovic et al. ${ }^{5}$ reported the association of up to 32 GM members with very-low-density (VLDL) and high-density (HDL) subfractions. Positive correlations between a number of Clostridiales members with large particle size subfractions of HDL were elucidated. In other studies, focusing on total lipoproteins fractions, an increasing abundance of GM members affiliated to the Erysipelotrichaceae and Lachnospiraceae families have been linked to elevated levels of total cholesterol and low-density lipoproteins (LDL) ${ }^{10-12}$. Interestingly, common gut microbes like Lactobacillaceae members have been reported to assimilate and lower cholesterol concentrations from growth media and incorporate it into their cellular 
Castro-Mejía et al.,

membrane ${ }^{15}$, whereas butyrate-producing Roseburia intestinalis has been found to increase fatty acid utilization and reduce atherosclerosis development in a murine model ${ }^{16}$. However, the relationship between GM and LPD distribution is still far from being understood. Thus, with the aim of gaining a deeper understanding of the relationship between GM and LPD in blood, we carried out a detailed compositional analysis of GM, its metabolic functions, and studied its associations with blood lipoproteins quantified using a recently developed method based on proton $\left({ }^{1} \mathrm{H}\right)$ nuclear magnetic resonance (NMR) spectroscopy ${ }^{6}$. We determined covariations between larger HDL subclasses and lower total cholesterol with a several Clostridiales (Ruminococcaceae and Lachnospiraceae) and Eggerthelalles members, whose metabolic potential is linked to biosynthesis of cofactors essential for carrying out lipid metabolism.

\section{METHODS}

\section{Study participants}

Two hundred and sixty-two men and women participants older than 20 years, who had not received antibiotic treatment 3 months prior to the beginning of the study and who had not received pre- or probiotics 1 month prior to the beginning of the study, were included as part of the COUNTERSTRIKE (COUNTERacting Sarcopenia with proTeins and exeRcise - Screening the CALM cohort for IIpoprotein biomarKErs) project (counterstrike.ku.dk). Pregnant and lactating women, as well as individuals suffering from CVD, diabetes or chronic gastrointestinal disorders, were excluded from the study.

\section{Ethics approval and consent to participate}

The study was approved by the Research Ethics Committees of the Capital Region of Denmark in accordance with the Helsinki Declaration (H-15008313) and the Danish Data 
Castro-Mejía et al.,

110 Protection Agency (2013-54-0522). Written informed consent was obtained from all

111 participants.

112

\section{Lipoprotein distribution profiles}

114 The human blood plasma lipoproteins were quantified using SigMa LP software ${ }^{17}$.

115 The SigMa LP quantifies lipoproteins from blood plasma or serum using optimized partial

116 least squares (PLS) regression models developed for each lipoprotein variable using one-

117 dimensional (1D) ${ }^{1} \mathrm{H}$ NMR spectra of blood plasma or serum and ultracentrifugation based

118 quantified lipoproteins as response variables as determined in Khakimov et al. ${ }^{6}$.

\section{Short chain fatty acids (SCFAs) quantification}

121 Targeted analysis and quantification of SCFA on fecal slurries were carried out as recently

122 described ${ }^{18}$

Samples processing, library preparation and DNA sequencing

Fecal samples were collected and kept at $4^{\circ} \mathrm{C}$ for maximum $48 \mathrm{~h}$ after voidance and

126 stored at $-60^{\circ} \mathrm{C}$ until further use. Extraction of genomic DNA and library preparation for

127 high-throughput sequencing of the V3-region of the 16S rRNA gene was performed as

128 previously described ${ }^{18}$. Shotgun metagenome libraries for sequencing of genome DNA were

129 built using the Nextera XT DNA Library Preparation Kit (Cat. No. FC-131-1096) and

130 sequenced with Illumina HiSeq 4000 by NXT-DX.

131

132 Analysis of sequencing data

133 The raw dataset containing pair-end amplicon reads we analyzed following recently

134 described procedures ${ }^{18}$. The metabolic potential of the amplicon sequencing dataset was

135 determined through PICRUSt ${ }^{19}$, briefly, zero-radious operational taxonomical units (zOTUs) 
Castro-Mejía et al.,

136 abundances were first normalized by copy number and then KEGG orthologues was obtained

137 by predicted metagenome function.

138 For shotgun sequencing, the reads were trimmed from adaptors and barcodes and the

139 high-quality sequences ( $>99 \%$ quality score) using Trimmomatic v0.35 ${ }^{20}$ with a minimum

140 size of 50nt were retained. Subsequently, sequences were dereplicated and check for the

141 presence of Phix179 using USEARCH v10 ${ }^{21}$, as well as human and plant genomes associated

142 DNA using Kraken2 ${ }^{22}$. High-quality reads were then subjected to within-sample de-novo

143 assembly-only using Spades v3.13.1 ${ }^{23}$ and the contigs with a minimum length of $2,000 \mathrm{nt}$

144 were retained. Within-sample binning was performed with metaWRAP ${ }^{24}$ using Metabat1 ${ }^{25}$,

145 Metabat2 ${ }^{26}$ and MaxBin2 ${ }^{27}$, and bin-refinement ${ }^{28}$ was allowed to a $\leq 10 \%$ contamination and

$146 \geq 70 \%$ completeness. Average nucleotide identity (ANI) of metagenome bins, or metagenome

147 assembled genomes (MAGs), was calculated with fastANI ${ }^{29}$ and distances between MAGs

148 were summarized with bactax ${ }^{30}$. To determined abundance across samples, reads were

149 mapped against MAGs with Subread aligner ${ }^{31}$ and a contingency-table of reads per Kbp of

150 contig sequence per million reads sample (RPKM) was generated. Taxonomic annotation of

151 MAGs was determined as follows: ORF calling and gene predictions were performed with

152 Prodigal ${ }^{32}$, the predicted proteins were blasted (blastp) against NCBI NR bacterial and

153 archaeal protein database. Using Basic Sequence Taxonomy Annotation tool (BASTA) ${ }^{33}$, the

154 Lowest Common Ancestor (LCA) for every MAG was estimated based on percentage of hits

155 of LCA of 60 , minimum identity of 0.7 , minimum alignment of 0.7 and a minimum number

156 of hits for LCA of 10.

157 To determine the metabolic potential of metagenomes, ORF calling and gene

158 predictions (similar as above) were performed on both, binned and unbinned contigs, and the

159 predicted proteins were subsequently clustered at 90\% similarity using USEARCH v10. To

160 assign functions, protein sequences were blasted (90\% id and 90\% cover query) against the

161 integrated reference catalog of the human gut microbiome (IRCHGM) ${ }^{34}$, while using only

162 target sequences containing KEGG ortholog entries. Similar as above, to determine 
Castro-Mejía et al.,

163 abundance of protein-encoding genes across metagenomes, reads were mapped against

164 protein clusters (PC) with DIAMOND ${ }^{35}$ and a contingency-table of reads mapped to PCs was

165 also generated. To avoid bias due to sequencing depth across protein-encoding genes, samples

166 were subsampled to $15,000,000$ reads per sample.

167

168

169

170

171

172

173

174

175

176

177

178

179

180

181

182

183

184

185

186

187

188

\section{Statistical analysis}

Stratification and clustering of LPD was carried out using Euclidean distances and general agglomerative hierarchical clustering procedure based on "Ward2", as implemented in the gplots R-package ${ }^{36}$. For univariate data analyses, pairwise comparisons were carried out with unpaired two-tailed Student's $t$-test, Spearman's rank coefficient was used for determining correlations and Chi-Square test for evaluating group distributions. For multivariate data analyses, the association of covariates (e.g. age, BMI, sex) with LPD were assessed by redundancy analysis (RDA) (999 permutations), whereas the association of LPD clusters with GM were analyzed by distance-based RDA (999 permutations) on Canberra distances (implemented in the vegan $\mathrm{R}$-package ${ }^{37}$ ).

Feature selection for zOTUs was performed with Random Forest. Briefly, for a given training set (training: 70\%, test: 30\%), the party R-package ${ }^{38}$ was run for feature selection using unbiased-trees (cforest_unbiased with 6,000 trees and variable importance with 999 permutations) and subsequently the selected variables were used to predict $(6,000$ trees with 999 permutations) their corresponding test set using randomForest $\mathrm{R}$-package ${ }^{39}$. The selected features were subjected to sequential rounds of feature selection until prediction could no longer be improved. All statistical analyses were performed in $\mathrm{R}$ versions $\leq 3.6 .0$.

\section{Data availability}

Sequence data are available at the Sequence Read Archive (SRA), BioProject SUB9304449 submissions SUB9305011 and SUB9304442. Supplementary Table 1 provides 
Castro-Mejía et al.,

189

190

samples information. Non-sequence data that support the findings of this study are available from the corresponding authors upon reasonable request.

\section{RESULTS}

\section{Participants and data collection}

Two hundred and sixty two individuals (men:women 90:172) with an age between 20 and 85 years (Figure 1A) and BMI ranging between 19 and $37 \mathrm{~kg} / \mathrm{m}^{2}$ (Figure 1B) were included in this study. Subjects are representatives of community dwelling and apparently healthy adults living in the Danish Capital Region. In this study, we included ${ }^{1} \mathrm{H}$ NMR spectroscopy based quantified lipoproteins from human blood plasma ${ }^{6}$, short-chain fatty acids profiling and GM composition on fecal samples based on 16S rRNA-gene amplicon sequencing and shotgun metagenome sequencing for a subset of samples (Figure 1C).

\section{LPD profiles, stratification and host covariates}

LPD profiles of the study subjects were predicted from ${ }^{1} \mathrm{H}$ NMR measurements of blood plasma. A total of 55 lipoproteins-subfractions were quantified including cholesterol, triglycerides (TG), cholesterol ester (CE), free cholesterol, phospholipids, apolipoprotein A (ApoA1) and apolipoprotein B (ApoB) content in all or in some of lipoprotein in plasma (VLDL, IDL, HDL, LDL) and/or in lipoprotein subfractions (HDL2a, HDL2b, HDL3, LDL1, LDL2, LDL3, LDL4, LDL5, LDL6) ${ }^{6}$. Linking host covariates and LPD profiles, redundancy analysis (RDA) of LPD profiles showed a significant $(p \leq 0.01)$ effect of age, BMI and sex on LPD profiles (Figure 2B) with a combined size effect of up to $24.6 \%$ (Figure $2 \mathrm{~B}-\mathrm{C}$ ). Clustering of LPD profiles segregated study participants into three groups (Figure 2A, Figure I in the Data Supplement). Cluster 1A and 1B were characterized by higher concentrations of LDL sub-fractions and their constituents (particularly evident in subclasses 1 and 2). Clusters $1 \mathrm{~A}$ and 2, on the other hand, were characterized by lower concentrations of 
Castro-Mejía et al.,

214 HDL sub-fractions (associated with HDL2a and HDL2b), whereas higher concentrations of

215 HDL-3 particles in subjects of cluster 1A were observed (Figure I in the Data Supplement).

216 Furthermore, plasma concentrations of CE, phospholipids and CE were higher among cluster

$2171 \mathrm{~A}$ and $1 \mathrm{~B}$. When comparing the plasma fractions of the study participants to the

218 recommendations of cholesterol classes provided by the National Institute of Health (NIH) ${ }^{40}$,

219 for clusters 1A and 1B total cholesterol and LDL levels were above the recommendations,

220 while for clusters 1B and 2 the levels pf HDL were below the recommended values.

221 LPD profiles were also found to covariate with host attributes, cluster 2 subjects was

222 significantly younger than clusters $1 \mathrm{~A}$ and $1 \mathrm{~B}$ (Figure 2D), and cluster 1B showed the lowest

223 BMI (Figure 2E). These results were also consistent even after correcting for sex effects,

224 given that cluster 1B had a significantly higher proportion of women (Fisher test $p<0.01$,

225 Figure 2A) compared to clusters 1A and 2 (Figure I in the Data Supplement).

\section{LPD clusters are linked with GM profiles}

The GM of study participants $(\mathrm{n}=262)$ was profiled using high-throughput amplicon sequencing the V3-region of the 16S rRNA gene (11,544 zOTUs), as well as shotgun metagenome sequencing of total genomic DNA for a subset of samples $(\mathrm{n}=58)$. Gene

231 content and functionality (based on KEGG orthologues - KOs) were predicted based on

232 PICRUSt ${ }^{19}$ (for 16S rRNA gene amplicons), as well as through ORF calling and gene

233 prediction of assembled contigs reconstructed from shotgun metagenome data. Validation of

234 PICRUSt against metagenome calling KO yielded a high correlation coefficient (Pearson $r=$

2350.77 , Figure $3 \mathrm{~A}$ ) between the gene richness of both datasets. Alpha diversity analyses

236 between LPD clusters revealed no significant $(t$-test $p>0.05)$ differences in phylotypes

237 (Figure 3B) nor KOs richness as predicted by the PICRUSt (Figure 3C). A significant (Dip-

238 test $p<0.001$ ) bimodal distribution of KO richness among the study participants was

239 observed, but a higher-/lower- gene count was not associated to LPD clusters (Figure 3C) or

240 BMI categories (Figure 3D). Significant differences in composition (beta-diversity) between 
Castro-Mejía et al.,

241 LPD clusters were observed among phylotypes (Canberra distance, Adonis test $p<0.05, \mathrm{R}^{2}=$ $2420.62-1 \%$ ), but not among PICRUSTs predicted KOs.

\section{LPD clusters correspond with GM and KOs features}

After feature selection based on random forest, LPD clusters were partially

246 discriminated (Figure 4A) by 206 selected sequence variants (zOTUs) distributed to over 10

247 families (Figure 4B). Among these, zOTUs affiliated to Ruminococcaceae (75) and

248 Lachnospiraceae (58) represented 64\%, followed by Bacteroidaceae (8), Bifidobacteriaceae

249 (7), Christensenellaceae (6), Coriobacteriaceae (5) and four other sparse bacterial families

250 (47). The cumulative abundance (cumulative sum scaling, CSS) of those families showed

251 differences between LPD clusters, with cluster 1A being associated with a higher abundance

252 of Lachnospiraceae and a lower abundance of Christensenellaceae members, while cluster 1B

253 was characterized by a larger proportion of Ruminococcaceae phylotypes, and cluster 2

254 showed increased proportion of Bifidobacteriaceae, Bacteroidaceae and reduced abundance of

255 Coriobacteriaceae (Figure 4B-C).

$256 \quad$ KEGG orthologues predicted through PICRUSt demonstrated very weak

257 discrimination power towards LPD clusters (Figure 4D, Figure II-A in the Data Supplement

258 shows detailed $3^{\text {rd }}$ level KEGG functions), this included 54 KOs affiliated to $>9$ primary and

259 secondary metabolism processes, as well as signaling and cellular processes (Figure 4E).

260 Despite its documented limitations ${ }^{41}$ PICRUSt was still able to reveal a decreasing

261 abundance of functional modules among subjects of cluster 1A and 2 as compared to those of

262 cluster 1B (Figure 4E-F). Analysis on aggregated functions per KOs ( $2^{\text {nd }}$ level KEGG)

263 showed that cluster 1B was characterized by a higher abundance (t-test $p<0.05$ ) of functions

264 related to metabolism of amino acids (e.g., Phe, Tyr and Trp biosynthesis), carbohydrates

265 (e.g., pyruvate, propanoate and butanoate metabolism), lipids (glycerolipids and

266 glycerophospholipids metabolism) and genetic information processing (e.g., transcriptional

267 factors) (Figure 4F). 
Castro-Mejía et al.,

269 (Spearman FDR $\mathrm{p} \leq$ 0.05) associations (Figure 4G, Figure II-B in the Data Supplement).

270 Most Ruminococcaceae (74/75 phylotypes, mostly unclassified), a division of

271 Lachnospiraceae (13/58 phylotypes, mostly unclassified), Bacteroidaceae (e.g., $B$.

272 massiliensis, B. caccae) Christensenellaceae (unclassified genus) and Coriobacteriaceae

273 (unclassified genus) showed positive correlations with HDL subfractions and negative

274 correlations with VLDL and LDL (e.g. LDL3, 4, 5 ,6). Contrary to this, most

275 Lachnospiraceae (45/58), Veillonellaceae (e.g., V. invisus) and Bifidobacteriaceae (e.g., $B f$.

276 adolescentis, Bf. bifidum) phylotypes correlated negatively with HDL subfractions, and

277 positively with subfractions composed of IDL, LDL and VLDL. For KOs vs LPD (Figure 4H,

278 Figure II-C in the Data Supplement), increasing abundance of functions linked to

279 glycerophospholipids metabolism and amino acids (His, Phe, Tyr and Trp) biosynthesis

280 correlated positively with HDL fractions and negatively with LDL and VLDL. Furthermore,

281 the production of glycosphingolipids, biotin ( $\left.\mathrm{Vit}_{\mathrm{B} 7}\right)$ and lipopolysaccharides correlated

282 negatively with small LDL subfractions (e.g. LDL3, 4, 5,6 ).

\section{Metagenome bins and functions associated with LPD clusters}

Fifty-eight samples were subjected to shotgun metagenome sequencing (Figure 1C) generating on average $5.2 \mathrm{~GB}$ per sample. ORF calling on the entire assembled dataset of generated $\sim 1.4$ million gene-clusters ( $90 \%$ similarity clusters, here termed "genes"), with 84,560 core genes being present in at least $90 \%$ of the metagenome sequenced samples. RDA analysis of the core-gene dataset showed significant $(p=0.001)$ differences between LPD clusters and explaining up to $23.7 \%$ of the total variance in gene composition (Figure 5A).

291 Ranking of variables (i.e. top 150) within the $1^{\text {st }}$ and $2^{\text {nd }}$ canonical components of the CAP 292 analyses provided an overview of 35 "known" metabolic genes ( $>90 \%$ identity match to the 293 integrated non-redundant gene catalog with KEGG ortholog entries ${ }^{34}$, Figure 5B, Figure III294 A in the Data Supplement) linked to $>102^{\text {nd }}$ level KEGG functions, which resembled the 
Castro-Mejía et al.,

large majority of those predicted by PICRUSt (see Figure 4E-F). A higher abundance of these genes was observed among subjects grouped within Cluster 1B relative to cluster 1A and Cluster 2. To determine the species associated with these genes, gene-sequences were mapped back to 1,419 metagenome-assembled genomes (MAGs) (Figure 5C). Sixty MAGs affiliated to Lachnospiraceae, Clostridiales, Coriobacteriaceae and Firmicutes and clustered within 19 species were found to contribute with 27 out of the 35 genes that discriminated LPD clusters (Figure 5D, Figure III-B in the Data Supplement). MAGs-G1 to G5 contributed with peptidoglycan and glycan biosynthesis. MAGs-G6 to G12 contributed with thiamine (Vit ${ }_{\text {B1 }}$ ) and pantothenate $\left(\mathrm{Vit}_{\mathrm{B} 5}\right)$ metabolism, starch degradation and butyric acid metabolism (butanol dehydrogenase that may lead to increased concentrations of 1-butanol at the expense of butyrate production, Figure 5E) and glycerolipid metabolism. Finally, MAGs-G13 to G19 promoted biosynthesis of glucosinates, metabolism of propionic acid, biosynthesis of fatty acids, $\mathrm{Vit}_{\mathrm{B} 6}$ metabolism, as well as folate (Vit ${ }_{\mathrm{B} 9}$ ) biosynthesis (Figure 5D, 5F, Figure III-B in the Data Supplement). Subjects belonging to LPD-cluster 1B had a significantly higher relative abundance of MAGs-G7, MAGs-G9 to G19 (those comprising Clostridiales, Eggerthellaceae and Firmicutes bins, Figure 5G-H), MAGs-G1 and MAGs-G5 (those affiliated to Lachnospiraceae, Figure 5I) than subjects in clusters 1A and 2. Likewise, their cumulative abundance reached significant positive (spearman $p<0.001$ ) correlations with constituents (e.g., Cholesteryl ester) of larger HDL sub-classes (HDL2a and HDL2b) (Figure $5 \mathrm{~J})$.

The concentrations of the SCFAs acetate and propionate in fecal samples showed no differences between LPD clusters. However, higher concentrations of butyrate, isobutyrate, 2methylbutyrate, valerate and isovalerate (ANOVA Tukey's HSD $p<0.05$ ) were observed in cluster 2 (Figure 6A-D). To determine whether microbial activity was linked to the production of such branched-chain fatty-acids, we then focused on analyzing the abundance of isobutyrate kinase (Figure IV-C in the Data Supplement) and 2-methylbutanoyl-CoA (Figure 6F) dehydrogenase in the metagenomic samples (Figure 6E-F). For 2- 
Castro-Mejía et al.,

methylbutanoyl-CoA dehydrogenase $86 \%$ of the gene-variants were also mapped to those 60 MAGs displayed in Figure 6F (ANOVA Tukey's HSD $p<0.05$ for cluster 2 LPD subjects), but none of these had significant matches to isobutyrate kinase. Isobutyrate kinase was found in 86 MAGs (Figure IV-A in the Data Supplement) belonging to Bacteroides, Ruminococcaceae, Alistipes, Desulfovibrionaceae and Lachnospiraceae, and whose cumulative relative abundance varied (Figure IV-B in the Data Supplement) substantially between LPD clusters.

\section{Discussion}

It is well established that certain LPD profiles are associated with elevated CVD risk, but relatively little is known on the links between GM and LPD. Building on recently published LPD profiles of 262 adult individuals ${ }^{6}$ the present study investigates the correlations between LPD-profiles and GM, and its genetic functional assignments. Stratification of study participants based on their LPD profiles yielded three LPD clusters (1A, 1B and 2) that corresponded well with within- and outside- suggested levels of total cholesterol, triglycerides, LDL, HDL and VLDL as those recommended by the $\mathrm{NIH}^{40}$ and as shown in Figure 2A. Our study demonstrates that lower levels of total HDL are associated with a decrease in the concentration of large subfractions (e.g. HDL2a and HDL2b), while higher levels of LDL correspond with an increase in the concentration of large LDL subfractions (e.g. LDL1). Similarly, high levels of cholesterol corresponded with high levels of circulating levels of VLDL. As confirmed by our results and others, the LPD profiles are influenced by host factors like age, sex and BMI ${ }^{5,10}$. These components are able to explain up to $25 \%$ of the total variance in the LPD. To the best of our knowledge, this study represents the first to show the contribution of LPD subfractions to the collective levels of cholesterol, cholesterol-types and triglycerides, as well as recommendations among an age/BMI- diverse group of apparently healthy adults. 
Castro-Mejía et al., distinct LPD profiles (e.g., C1A, C1B and C2 clusters). Despite the fact that a bimodal

351 distribution of gene-richness counts was reproduced as in previous studies ${ }^{45,46}$ no significant

352 differences in the gene-frequencies between normal and overweight participants were 353 observed. particles (e.g., LDL3, LDL4 and LDL5), ILDL and VLDL, while Ruminococcaceae, a subgroup of Lachnospiraceae phylotypes and other less abundant families showed positive 358 correlations with large particles of HDL (HDL2a and HDL2b (see e.g., Figure 4G).

359 Moreover, in agreement with our findings, a recent large-scale study published by Vojinovic 360 et al. ${ }^{5}$ also reported that Lachnospiraceae and Ruminococcaceae members were related to the 361 HDL/LDL ratios. High HDL levels have been consistently correlated to a low risk of 362 developing $\operatorname{CVD}^{7,8}$ and recent evidence support that the heterogeneity of HDL display 363 different associations with the incidence of CVD and metabolic syndrome ${ }^{7,47,48}$. Recent 364 findings suggest that Akkermansia muciniphila induces expression of low-density lipoprotein 365 receptors and ApoE in the hepatocytes, facilitating the clearance of triglyceride-rich 366 lipoprotein remnants, chylomicron remnants, and intermediate-density lipoproteins, from 367 circulation ${ }^{42}$. In line with this, our study elucidates a possible link between dyslipidemia and 368 the metabolic potential of MAGs for biosynthesizing important bioactive compounds such as 369 vitamin B complex and peptidoglycans, as well as SCFA metabolism. Among these 370 compounds, pantothenate $\left(\mathrm{Vit}_{\mathrm{B}}\right)$, $\mathrm{Vit}_{\mathrm{B} 6}$ and folate ( $\left.\mathrm{Vit}_{\mathrm{B} 9}\right)$ have been inversely associated with 371 low-grade inflammation ${ }^{49}$ and mortality risk of CVD in a mechanism that may involve 372 regulation of blood homocysteine concentrations ${ }^{50}$ and one-carbon metabolism ${ }^{51}$. SCFA like 373 butyrate and valerate have been shown to decrease total cholesterol and the expression of 
Castro-Mejía et al.,

mRNA associated with fatty acid synthase and sterol regulatory element binding protein 1c, to enhance mRNA expression of carnitine palmitoyltransferase- $1 \alpha(\mathrm{CPT}-1 \alpha)$ in liver ${ }^{52,53}$, as well as to ameliorate arteriosclerosis via ABCA1-mediates cholesterol efflux in macrophages

${ }^{54}$. Biosynthesis of peptidoglycans by some GM members has been associated with incidence of stenotic atherosclerotic plaques and insulin resistance ${ }^{55,56}$. However, emerging evidence suggests that these potent signaling molecules play positive roles for enhancing systemic innate immunity ${ }^{57}$ and neurodevelopmental processes ${ }^{58}$, relaying on a species-dependent fashion ${ }^{59}$. In conclusion, our study provides evidence that GM members (e.g., MAGs) and their genes related to the biosynthesis of bioactive molecules needed to carry out lipid metabolism, e.g., vitamin B complex and S/B-CFA, are more abundant among subjects with healthier LPD profiles (e.g., higher HDL2a, HDL2b, and lower LDL). Furthermore, variations in LPD subfractions correlates with differences in the GM composition ${ }^{5}$, but these are not necessarily associated to a higher or lower microbial diversity as reported in previous studies ${ }^{45,46}$. Given the cross-sectional nature of our study and its inherent limitations, it is not possible to depict the mechanism by which GM may influence variability in LPD subfractions. However, our results provide evidence for GM considerations in future research aiming at unravelling the processes of LPD particles assembly through longitudinal mechanistic approaches that include the activity of enzymes and transfer proteins, membrane modulators ${ }^{60}$ and integrative multi-omics.

\section{ARTICLE INFORMATION}

\section{Affiliations}

Department of Food Science, University of Copenhagen (JLC, BK, EG, PP, SBE, DSN).

Department of Nutrition, Exercise and Sports, University of Copenhagen (MVL). Department 
bioRxiv preprint doi: https://doi.org/10.1101/2021.09.01.458531; this version posted September 2, 2021. The copyright holder for this

preprint (which was not certified by peer review) is the author/funder, who has granted bioRxiv a license to display the preprint in perpetuity. It is made available under aCC-BY-NC-ND 4.0 International license.

Castro-Mejía et al.,

399 of Biomedical Sciences, University of Copenhagen (EG, LH). Institute of Experimental

400 Endocrinology, Biomedical Research Center, University Science Park for Biomedicine,

401 Slovakia (PP). Swammerdam Institute for Life Sciences, University of Amsterdam (ET, AS).

402 Department of Plant and Environmental Sciences, University of Copenhagen (LHH). School

403 of Sport, Exercise and Rehabilitation Sciences, University of Birmingham (LH).

404

405

Source of Funding

406

The present study received funding from the Innovation Foundation Denmark

407 through the COUNTERSTRIKE project (4105-00015B).

408

409

Disclosures

410 None.

411

412 Supplemental Materials

413 Data Supplement Figures I - IV

414 Supplementary-table_1

415

416

417

418

419

420

421

422

423

424

425 
Castro-Mejía et al.,

\section{References}

430 1. Zhang H, Temel RE, Martel C. Cholesterol and lipoprotein metabolism: Early career

431 committee contribution. Arterioscler Thromb Vasc Biol 2014;34:1791-1794. doi:10.1161/ATVBAHA.114.304267.

2. Vallejo-Vaz AJ, Robertson M, Catapano AL, Watts GF, Kastelein JJ, Packard CJ, Ford I, Ray KK. Low-Density Lipoprotein Cholesterol Lowering for the Primary Prevention of Cardiovascular Disease Among Men With Primary Elevations of Low-Density Lipoprotein Cholesterol Levels of $190 \mathrm{mg} / \mathrm{dL}$ or Above: Analyses From the WOSCOPS (West of Scotland Coronary Prevention Study) 5-Year Randomized Trial and 20-Year Observational Follow-Up. Circulation 2017;136:1878-1891. doi:10.1161/CIRCULATIONAHA.117.027966.

3. Nelson ER, Chang C yi, McDonnell DP. Cholesterol and breast cancer pathophysiology. Trends Endocrinol Metab 2014;25:649-655. doi:10.1016/j.tem.2014.10.001.

4. Aru V, Lam C, Khakimov B, et al. Quantification of lipoprotein profiles by nuclear magnetic resonance spectroscopy and multivariate data analysis. TrAC - Trends Anal Chem 2017;94:210-219. doi:10.1016/j.trac.2017.07.009.

5. Vojinovic D, Radjabzadeh D, Kurilshikov A, et al. Relationship between gut microbiota and circulating metabolites in population-based cohorts. Nat Commun 2019;10:5813. doi:10.1038/s41467-019-13721-1.

6. Khakimov B, Hoefsloot HCJ, Mobaraki N, et al. Human blood lipoprotein predictions from 1 H NMR spectra: protocol, model performances and cage of covariance. BioRxiv 2021.02.24.432509. doi: https://doi.org/10.1101/2021.02.24.432509

7. Holmes M V., Millwood IY, Kartsonaki C, et al. Lipids, Lipoproteins, and Metabolites and Risk of Myocardial Infarction and Stroke. J Am Coll Cardiol 2018;71:620-632. doi:10.1016/j.jacc.2017.12.006.

8. Wang J, Stančáková A, Soininen P, Kangas AJ, Paananen J, Kuusisto J, Ala-Korpela M, Laakso M. Lipoprotein subclass profiles in individuals with varying degrees of glucose tolerance: A population-based study of 9399 Finnish men. J Intern Med 2012;272:562-572. doi:10.1111/j.1365-2796.2012.02562.x.

9. Zheng D, Liwinski T, Elinav E. Interaction between microbiota and immunity in health and

459 10. Fu J, Bonder MJ, Cenit MC, et al. The gut microbiome contributes to a substantial proportion of the variation in blood lipids. Circ Res 2015;117:817-824. 
Castro-Mejía et al.,

doi:10.1161/CIRCRESAHA.115.306807.

11. Falony G, Joossens M, Vieira-Silva S, et al. Population-level analysis of gut microbiome variation. Science 2016;352:560-564. doi:10.1126/science.aad3503.

12. Rothschild D, Weissbrod O, Barkan E, et al. Environment dominates over host genetics in shaping human gut microbiota. Nature 2018;555:210-215. doi:10.1038/nature25973.

13. Koeth RA, Wang Z, Levison BS, et al. Intestinal microbiota metabolism of l-carnitine, a nutrient in red meat, promotes atherosclerosis. Nat Med 2013;19:576-585. doi:10.1038/nm.3145.

14. Le Roy T, Lécuyer E, Chassaing B, et al. The intestinal microbiota regulates host cholesterol homeostasis. BMC Biol 2019;17:94. doi:10.1186/s12915-019-0715-8.

15. Liong MT, Shah NP. Acid and bile tolerance and cholesterol removal ability of lactobacilli strains. J Dairy Sci 2005;88:55-66. doi:10.3168/jds.S0022-0302(05)72662-X.

16. Kasahara K, Krautkramer KA, Org E, et al. Interactions between Roseburia intestinalis and diet modulate atherogenesis in a murine model. Nat Microbiol 2018;3:1461-1471. doi:10.1038/s41564-018-0272-x.

17. Khakimov B, Mobaraki N, Trimigno A, Aru V, Engelsen SB. Signature Mapping (SigMa): An efficient approach for processing complex human urine $1 \mathrm{H}$ NMR metabolomics data. Anal Chim Acta 2020;1108:142-151. doi:10.1016/j.aca.2020.02.025.

18. Castro-Mejía JL, Khakimov B, Krych $\mathrm{E}$, et al. Physical fitness in community-dwelling older adults is linked to dietary intake, gut microbiota, and metabolomic signatures. Aging Cell 2020;19:e13105.

19. Langille MGI, Zaneveld J, Caporaso JG, et al. Predictive functional profiling of microbial communities using 16S rRNA marker gene sequences. Nat Biotechnol 2013;31:814-821. doi:10.1038/nbt.2676.

20. Bolger AM, Lohse M, Usadel B. Trimmomatic: A flexible trimmer for Illumina sequence data. Bioinformatics 2014;30:2114-2120. doi:10.1093/bioinformatics/btu170.

21. Edgar R. UNOISE2: improved error-correction for Illumina 16S and ITS amplicon sequencing. BioRxiv 2016:081257. doi:10.1101/081257.

22. Wood DE, Lu J, Langmead B. Improved metagenomic analysis with Kraken 2. Genome Biol 2019;20:257. doi:10.1186/s13059-019-1891-0.

23. Bankevich A, Nurk S, Antipov D, et al. SPAdes: A new genome assembly algorithm and its applications to single-cell sequencing. J Comput Biol 2012;19:455-477. doi:10.1089/cmb.2012.0021.

24. Uritskiy G V., Diruggiero J, Taylor J. MetaWRAP - A flexible pipeline for genome-resolved metagenomic data analysis. Microbiome 2018;6:158. doi:10.1186/s40168-018-0541-1.

25. Kang DD, Froula J, Egan R, Wang Z. MetaBAT, an efficient tool for accurately reconstructing single genomes from complex microbial communities. PeerJ 2015;3:e1165. doi:10.7717/peerj.1165.

26. Kang DD, Li F, Kirton E, Thomas A, Egan R, An H, Wang Zhong. MetaBAT 2: An adaptive 
Castro-Mejía et al., binning algorithm for robust and efficient genome reconstruction from metagenome

27. Wu Y-W, Tang Y-H, Tringe SG, Simmons BA, Singer SW. MaxBin: an automated binning assemblies. PeerJ 2019; 7:e7359. doi:10.7717/peerj.7359. method to recover individual genomes from metagenomes using. Microbiome 2014;2:26. doi.org/10.1186/2049-2618-2-26

28. Song WZ, Thomas T. Binning-refiner: Improving genome bins through the combination of different binning programs. Bioinformatics 2017;33:1873-1875.

29. Jain C, Rodriguez-R LM, Phillippy AM, Konstantinidis KT, Aluru S. High throughput ANI analysis of $90 \mathrm{~K}$ prokaryotic genomes reveals clear species boundaries. Nat Commun 2018;9:5114. doi:10.1038/s41467-018-07641-9.

30. Carroll LM, Wiedmann M, Kovac J. Proposal of a taxonomic nomenclature for the bacillus cereus group which reconciles genomic definitions of bacterial species with clinical and industrial phenotypes. MBio 2020;11: e00034-20. doi:10.1128/mBio.00034-20.

31. Liao Y, Smyth GK, Shi W. The Subread aligner: Fast, accurate and scalable read mapping by seed-and-vote. Nucleic Acids Res 2013;e108. doi:10.1093/nar/gkt214.

32. Hyatt D, Chen GL, LoCascio PF, Land ML, Larimer FW, Hauser LJ. Prodigal: Prokaryotic gene recognition and translation initiation site identification. BMC Bioinformatics 2010;11:119. doi:10.1186/1471-2105-11-119.

33. Kahlke T, Ralph PJ. BASTA - Taxonomic classification of sequences and sequence bins using last common ancestor estimations. Methods Ecol Evol 2019;10:100-103. doi:10.1111/2041210X.13095.

34. Li J, Wang J, Jia H, et al. An integrated catalog of reference genes in the human gut microbiome. Nat Biotechnol 2014;32:834-841. doi:10.1038/nbt.2942.

35. Buchfink B, Xie C, Huson DH. Fast and sensitive protein alignment using DIAMOND. Nat Methods 2015;12:59-60. doi:10.1038/nmeth.3176.

36. Warnes G, Bolker B, Bonebakker L, et al. gplots. R Packag 2020

37. Oksanen AJ, Blanchet FG, Kindt R, et al. vegan: Community Ecology Package. R Packag 2015.

38. Hothorn T, Hornik K, Strobl C, Zeileis A. Party: a laboratory for recursive partytioning. R Packag 2016.

39. Liaw A, Wiener M. Classification and Regression by randomFores. R Packag 2014.

40. National Insititute of Health (NIH). Detection, Evaluation, and Treatmentof High Blood Cholesterol in Adults (Adult TreatmentPanel III). NIH Publication 2002; 02-5215.

41. Sun S, Jones RB, Fodor AA. Inference-based accuracy of metagenome prediction tools varies across sample types and functional categories. Microbiome 2020;8:46. doi:10.1186/s40168020-00815-y. 
Castro-Mejía et al.,

Akkermansia muciniphila in Genetic-Induced Hyperlipidemia. Arterioscler Thromb Vasc Biol 2016;36:1448-1456. doi:10.1161/ATVBAHA.116.307597.

43. Kiouptsi K, Jäckel S, Pontarollo G, et al. The microbiota promotes arterial thrombosis in lowdensity lipoprotein receptor-deficient mice. MBio 2019;10: e02298-19. doi:10.1128/mBio.02298-19.

44. Rune I, Rolin B, Larsen C, et al. Modulating the gut microbiota improves glucose tolerance, lipoprotein profile and atherosclerotic plaque development in ApoE-deficient mice. PLoS One 2016;11: e0146439. doi:10.1371/journal.pone.0146439.

45. Le Chatelier E, Nielsen T, Qin J, et al. Richness of human gut microbiome correlates with metabolic markers. Nature 2013;500:541-546. doi:10.1038/nature12506.

46. Cotillard A, Kennedy SP, Kong LC, et al. Dietary intervention impact on gut microbial gene richness. Nature 2013;500:585-588. doi:10.1038/nature12480.

47. Kontush A. HDL particle number and size as predictors of cardiovascular disease. Front Pharmacol 2015;6:218. doi:10.3389/fphar.2015.00218.

48. Camont L, Chapman MJ, Kontush A. Biological activities of HDL subpopulations and their relevance to cardiovascular disease. Trends Mol Med 2011;17:594-603. doi:10.1016/j.molmed.2011.05.013.

49. Jung S, Kim MK, Choi BY. The long-term relationship between dietary pantothenic acid (vitamin B5) intake and C-reactive protein concentration in adults aged 40 years and older. Nutr Metab Cardiovasc Dis 2017;27:806-816. doi:10.1016/j.numecd.2017.05.008.

50. Cui R, Iso H, Date C, Kikuchi S, Tamakoshi A. Dietary folate and vitamin B6 and B12 intake in relation to mortality from cardiovascular diseases: Japan collaborative cohort study. Stroke 2010;41:1285-1289. doi:10.1161/STROKEAHA.110.578906.

51. Walker AK, Jacobs RL, Watts JL, et al. A Conserved SREBP-1/Phosphatidylcholine Feedback Circuit Regulates Lipogenesis in Metazoans. Cell 2011;147:840-852. doi:10.1016/j.cell.2011.09.045.

52. Nguyen TD, Prykhodko O, Fåk Hållenius F, Nyman M. Monovalerin and trivalerin increase brain acetic acid, decrease liver succinic acid, and alter gut microbiota in rats fed high-fat diets. Eur J Nutr 2019;58:1545-1560. doi:10.1007/s00394-018-1688-z.

53. Jiao AR, Diao H, Yu B, et al. Oral administration of short chain fatty acids could attenuate fat deposition of pigs. PLoS One 2018;13: e0196867. doi:10.1371/journal.pone.0196867.

54. Du Y, Li X, Su C, Xi M, Zhang X, Jiang Z, Wang L, Hong B. Butyrate protects against highfat diet-induced atherosclerosis via up-regulating ABCA1 expression in apolipoprotein Edeficiency mice. Br J Pharmacol 2020;177:1754-1772. doi:10.1111/bph.14933.

55. Karlsson FH, Fåk F, Nookaew I, Tremaroli V, Fagerberg B, Petranovic D, Bäckhed F, Nielsen $\mathrm{J}$. Symptomatic atherosclerosis is associated with an altered gut metagenome. Nat Commun 2012;3:1245. doi:10.1038/ncomms2266.

56. Denou E, Lolmède K, Garidou L, et al. Defective NOD 2 peptidoglycan sensing promotes 
bioRxiv preprint doi: https://doi.org/10.1101/2021.09.01.458531; this version posted September 2, 2021. The copyright holder for this

preprint (which was not certified by peer review) is the author/funder, who has granted bioRxiv a license to display the preprint in perpetuity. It is made available under aCC-BY-NC-ND 4.0 International license.

Castro-Mejía et al.,

578

579

580

581

582

583

584

585

586

587

588

589

590

591

592

593

594

595

596

597

598

599

600

601

602

603

604

605

606

607

608

609 diet-induced inflammation, dysbiosis, and insulin resistance . EMBO Mol Med 2015;7:259-

274. doi:10.15252/emmm.201404169.

57. Clarke TB, Davis KM, Lysenko ES, Zhou AY, Yu Y, Weiser JN. Recognition of peptidoglycan from the microbiota by Nod1 enhances systemic innate immunity. Nat Med 2010;16:228-231. doi:10.1038/nm.2087.

58. Tosoni G, Conti M, Diaz Heijtz R. Bacterial peptidoglycans as novel signaling molecules from microbiota to brain. Curr Opin Pharmacol 2019;48:107-113. doi:10.1016/j.coph.2019.08.003.

59. Baik JE, Jang YO, Kang SS, Cho K, Yun CH, Han SH. Differential profiles of gastrointestinal proteins interacting with peptidoglycans from Lactobacillus plantarum and Staphylococcus aureus. Mol Immunol 2015;65:77-85. doi:10.1016/j.molimm.2015.01.007.

60. Superko HR. Advanced Lipoprotein Testing and Subfractionation Are Clinically Useful.

Circulation 2009;119: 2383-2395. doi:10.1161/CIRCULATIONAHA.108.809582. 
bioRxiv preprint doi: https://doi.org/10.1101/2021.09.01.458531; this version posted September 2, 2021. The copyright holder for this

preprint (which was not certified by peer review) is the author/funder, who has granted bioRxiv a license to display the preprint in perpetuity. It is made available under aCC-BY-NC-ND 4.0 International license.

610

611

\section{FIGURE LEGENDS}

613
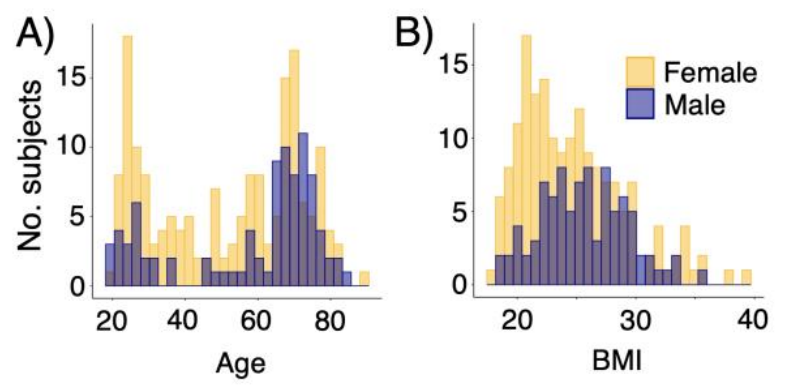

C) Host

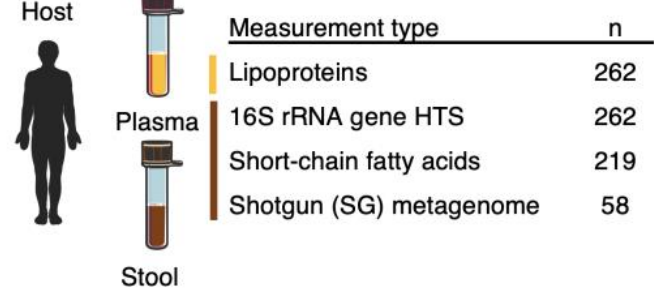

615

616

617

618

Figure 1. COUNTERSTRIKE participants and sample overview

Castro-Mejía et al.,

A) Age and B) body mass index (BMI) distribution of the study participants. C) samples and datasets included and analyzed in this study. 
bioRxiv preprint doi: https://doi.org/10.1101/2021.09.01.458531; this version posted September 2, 2021. The copyright holder for this preprint (which was not certified by peer review) is the author/funder, who has granted bioRxiv a license to display the preprint in perpetuity. It is made available under aCC-BY-NC-ND 4.0 International license.

Castro-Mejía et al.,

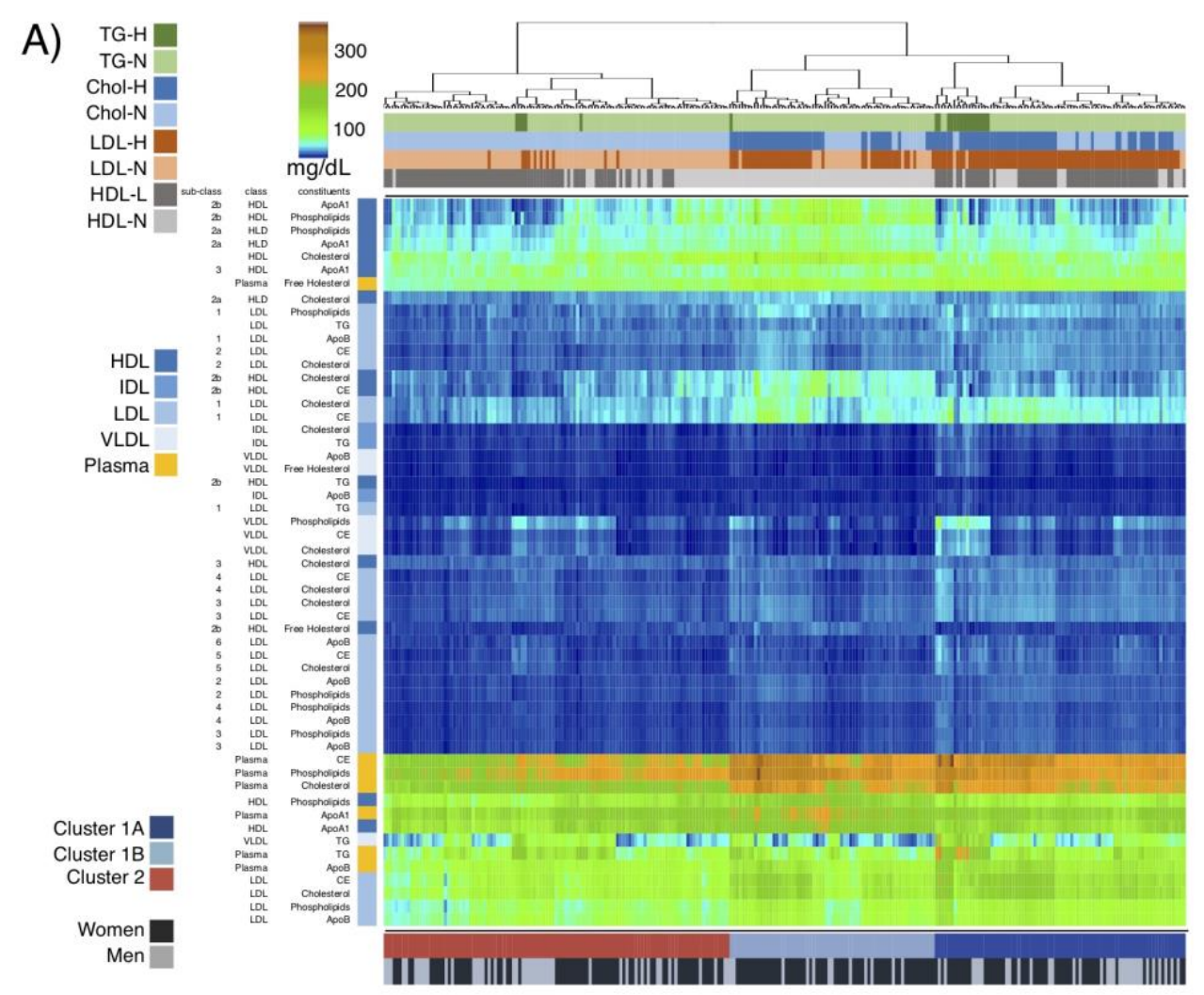

C)

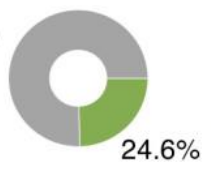

Unexplained Combined effect size

B)

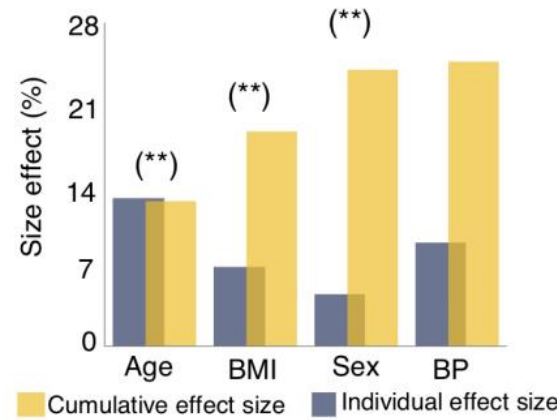

D)

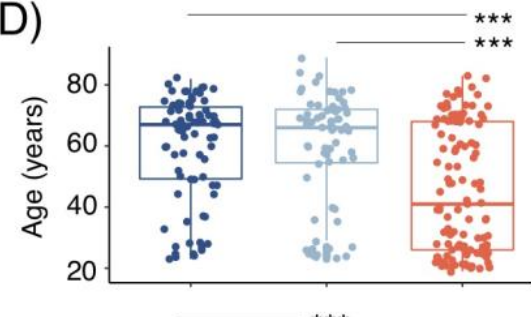

E)

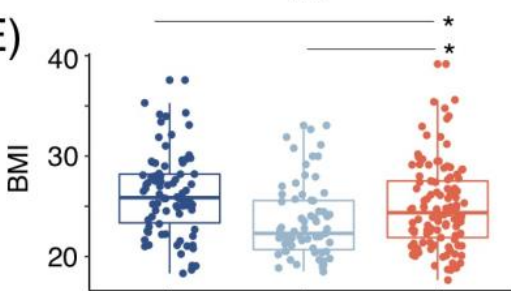

621 A) Profiles of main and sub-fractions of plasma lipoprotein distribution (LPD) determined by ${ }^{1} \mathrm{H}-\mathrm{NMR}^{6}$. LPD are clustered using Euclidean distances and general agglomerative hierarchical clustering procedure. Upper color bars represent within-/out- of the recommended levels of main lipoprotein fractions suggested by the $\mathrm{NIH}^{40}$ (total cholesterol $<200 \mathrm{mg} / \mathrm{dL}, \mathrm{LDL}<100 \mathrm{mg} / \mathrm{dL}, \mathrm{HDL}>60 \mathrm{mg} / \mathrm{dL}$, Triglycerides $<150 \mathrm{mg} / \mathrm{dL}$ ). Lower color bars depict 3 clusters (C1A, C1B and C2) of study participants given their LPD profile and the sex distribution of subjects. B) Cumulative effect size of non-redundant covariates of LPD determined by stepwise RDA analysis (right bars) as compared to individual effect sizes assuming independence (left bars). C) Fraction of LPD variation explained with the stepwise approach. Distribution of $\mathbf{D}$ ) age and $\mathbf{E}$ ) body mass index (BMI) between subjects belonging to $\mathrm{C} 1 \mathrm{~A}, \mathrm{C} 1 \mathrm{~B}$ and $\mathrm{C} 2$. Stars show statistical level of significance $\left({ }^{*} p \leq 0.05,{ }^{* *} p \leq 0.01, * * * P \leq\right.$ $0.001)$ 
Castro-Mejía et al.,

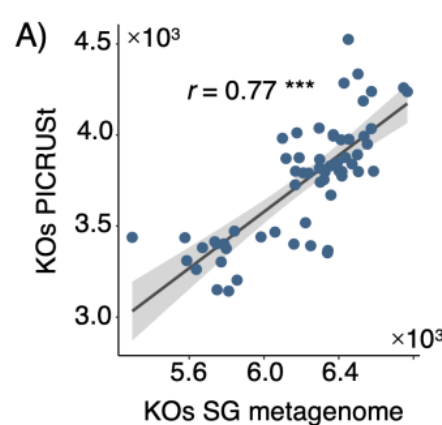

B)

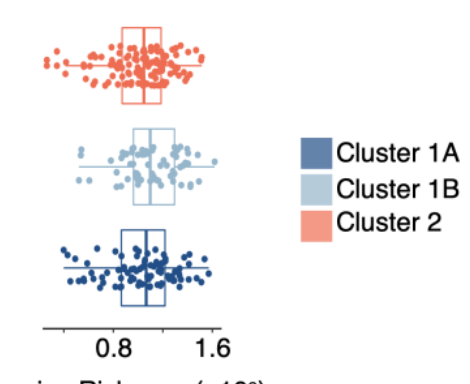

Species Richness $\left(\times 10^{3}\right)$

C)
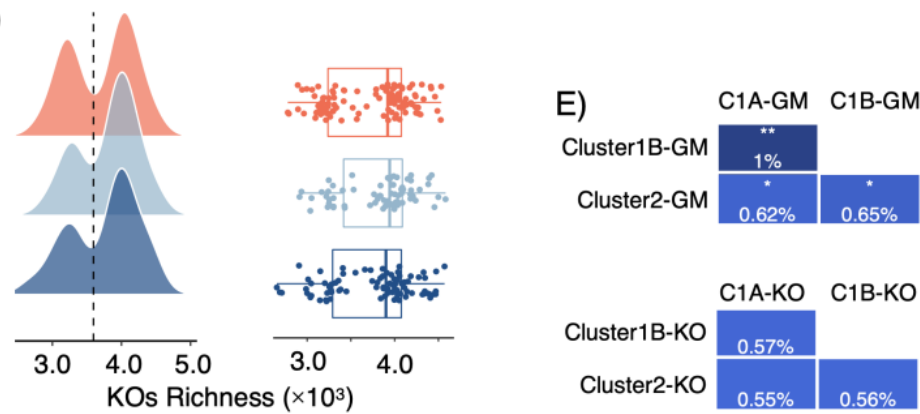

D)

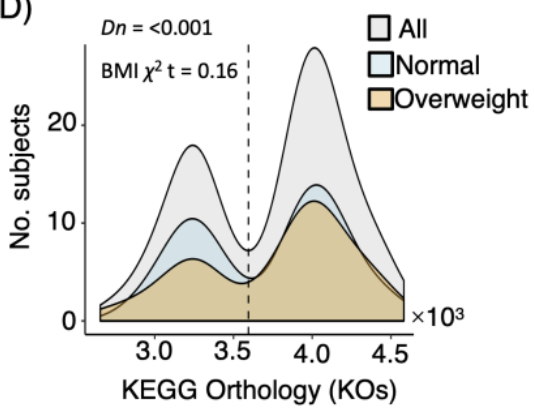

635

636

637

638

639

640

641

642

643

644

645

646

647

648

649

650

651

652

653

654

Figure 3. Diversity metrics on gut microbiota and metabolic content

A) Spearman's rank correlation between fecal microbial KEGG Orthologues (KOs) from shotgun metagenome (SG) sequencing and KO predicted by PICRUSt. B) Richness of microbial phylotypes (zOTUs) richness and $\mathbf{C}$ ) KO predicted by PICRUSt among subjects catalogued as being C1A, C1B and C2 based on their LPD. D) KO counts (richness) among all subjects and those with $\mathrm{BMI} \leq 25$ (normal) and $\mathrm{BMI}>25$ (overweighed); the observed bimodal distribution was statistically significant by the dip-test. E) Adonis test based on Canberra dissimilarities quantifying variance explained $\left(R^{2}\right)$ and significance of phylotypes and KO abundance with LPD clustering. Stars show statistical level of significance $\left(^{*} p \leq 0.05\right.$, $* * p \leq 0.01, * * * P \leq 0.001)$ 
bioRxiv preprint doi: https://doi.org/10.1101/2021.09.01.458531; this version posted September 2,2021 . The copyright holder for this preprint (which was not certified by peer review) is the author/funder, who has granted bioRxiv a license to display the preprint in perpetuity. It is made available under aCC-BY-NC-ND 4.0 International license.

Castro-Mejía et al.,

A)
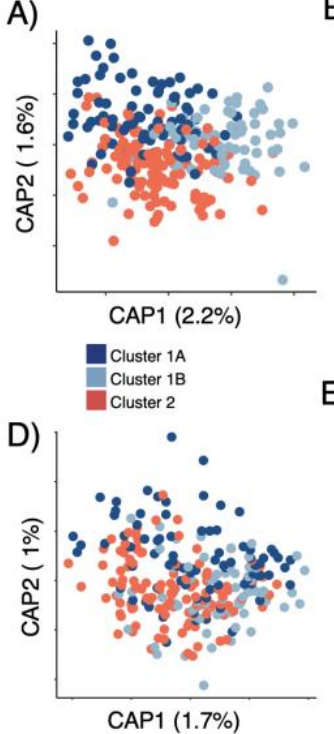

B)

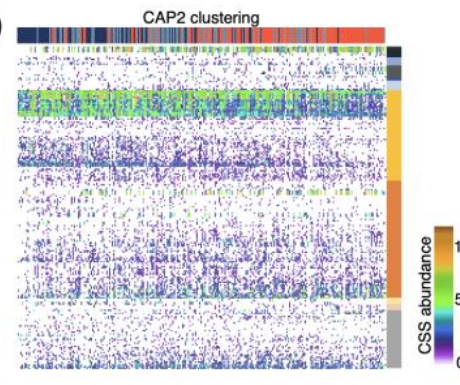

E)

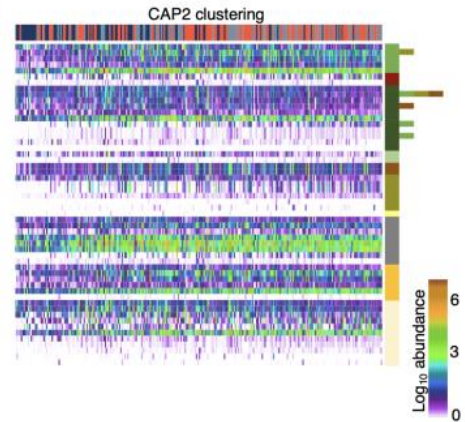

C)
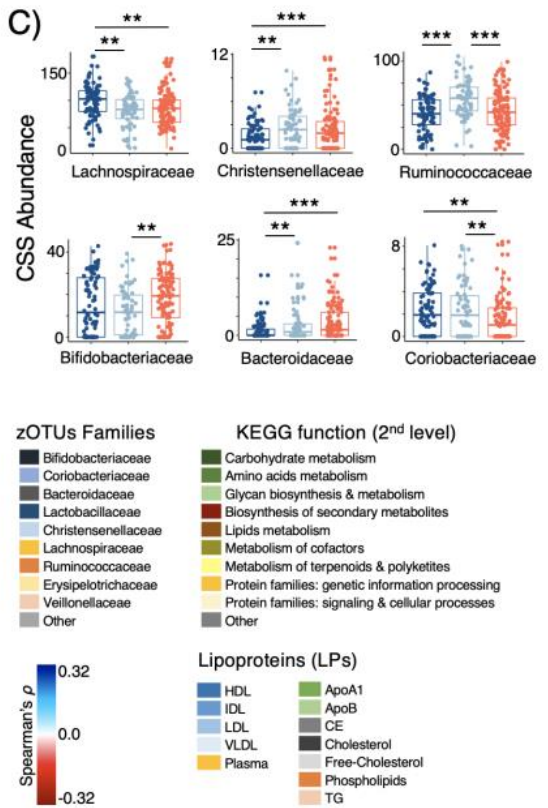

H)

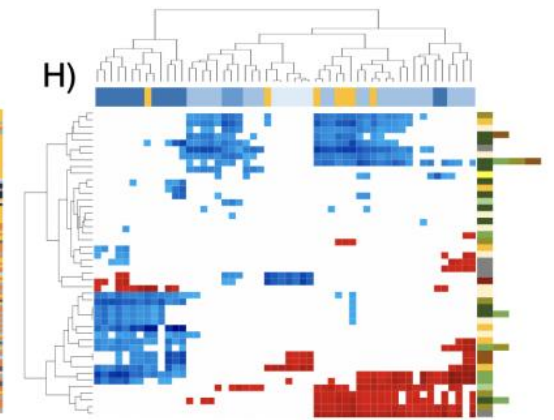

F)

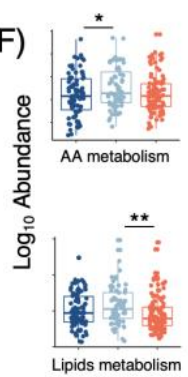

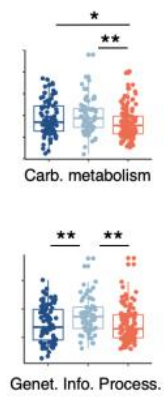

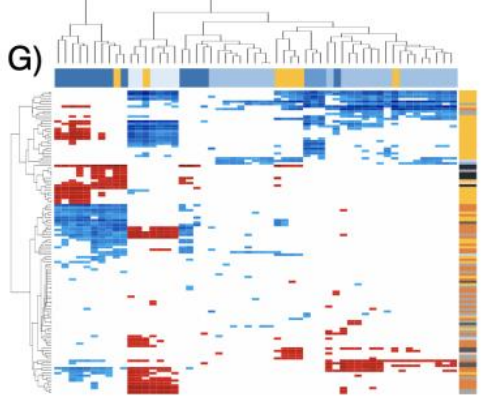

Figure 4. Phylotypes and KO functions associated with LPD clustering

Distance-based RDA (Canberra dissimilarity) displaying discrimination of LPD clusters based on selected $\mathbf{A})$ zOTUs $(p=0.001$, explained variance $=3.8 \%)$ and $\mathbf{D})$ KOs-PICRUSt $(p$ $=0.001$, explained variance $=2.7 \%$ ) selected through Random Forests. Overview of selected B) zOTUs and E) KOs-PICRUSt clustered using Canberra distances and general agglomerative hierarchical clustering procedure based on ward2. Distribution of $\mathbf{C}$ ) zOTUs summarized to family level and $\mathbf{F}$ ) KOs-PICRUSt summarized to $2^{\text {nd }}$ level KEGG function across subjects belonging C1 A, C1B and C2 LPD groups. Heatmaps displaying significant (False Discovery Rate corrected, FDR $\leq 0.05$ ) Spearman's rank correlations between $\mathbf{G}$ ) zOTUs and LPD sub-fractions, as well as $\mathbf{H}$ ) KOs-PICRUSt and LPD sub-fractions. Stars show statistical level of significance $\left({ }^{*} p \leq 0.05,{ }^{* *} p \leq 0.01, * * * P \leq 0.001\right)$ 
bioRxiv preprint doi: https://doi.org/10.1101/2021.09.01.458531; this version posted September 2, 2021. The copyright holder for this preprint (which was not certified by peer review) is the author/funder, who has granted bioRxiv a license to display the preprint in perpetuity. It is made available under aCC-BY-NC-ND 4.0 International license.

Castro-Mejía et al.,
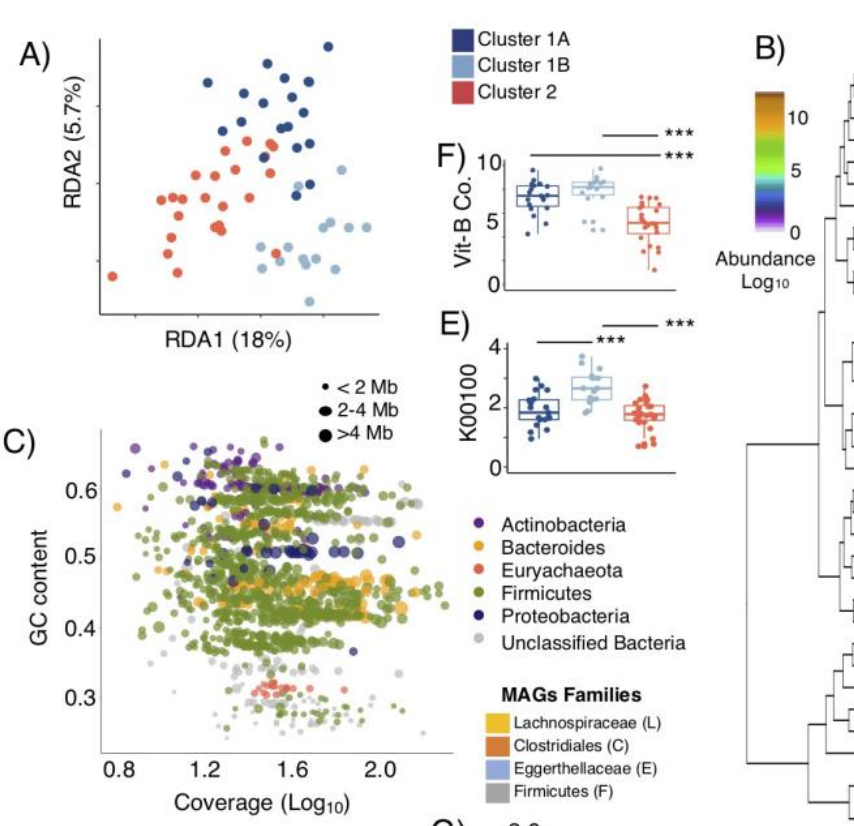

D)

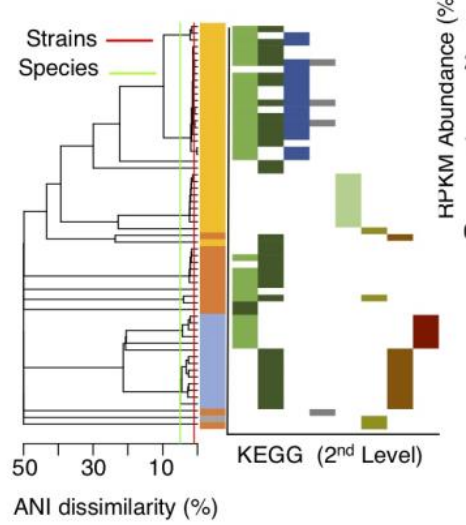

G)

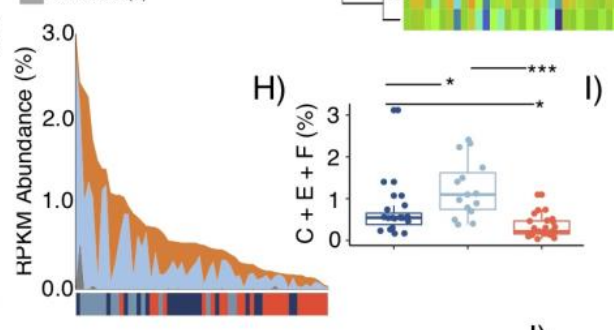

Actinobacteria

- Bacteroides

- Euryachaeota

- Proteobacteria

Unclassified Bacteria

MAGs Families $\quad$ Lachnospiraceae (L)
$\quad$ Clostridiales (C)
Eggerthellaceae (E) Firmicutes (F)
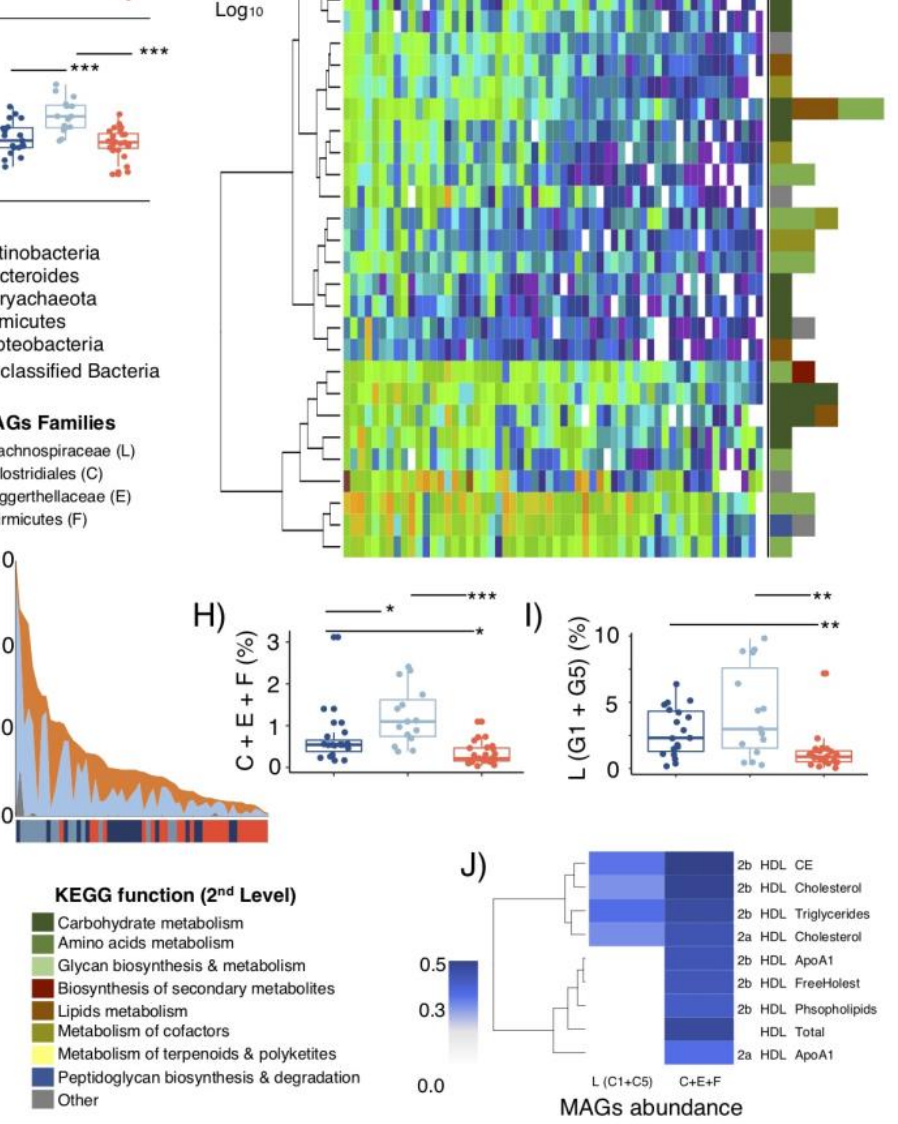

Figure 5. Metagenome metabolic functions and associated MAGs

A) RDA displaying discrimination of LPD clusters based on selected KOs obtained from shotgun metagenome and assembly $(p=0.001$, explained variance $=23.7 \%)$. B) Overview of most discriminatory (based on CAP1 and CAP2 within db-RDA) KOs with known metabolic functions clustered using Canberra distances and general agglomerative hierarchical clustering procedure based on ward2. C) GC-content - Coverage plot of metagenome assembled genomes (MAGs) with $\leq 10 \%$ contamination and $\geq 70 \%$ completeness. MAGs are colored according to phylum-level taxonomic affiliation and bubble size indicates their genome size in mega-bases (Mb). D) Phylogeny of MAGs containing KOs that discriminate LPD clusters (1A, 1B and 2), a cut-off of 95-ANI (species-level) and 99-ANI (strain-level) are denoted. MAGs are colored at family level affiliations and their KOs contribution at the $2^{\text {nd }}$ level KEGG function pathways are provided. E) Relative abundance of protein-encoding genes associated with butanol dehydrogenase (K00100), and F) protein-encoding genes associated metabolism and biosynthesis of vitamin B1, B2, B5 and B9. G-H) Distribution of cumulative abundance (RPKM) of MAGs (containing discriminatory KOs) associated with Clostridiales, Coriobacteriaceae and Firmicutes $(\mathrm{Cl}+\mathrm{Co}+\mathrm{F})$ among LPD clusters. I) Distribution of cumulative abundance (RPKM) of MAGs (G1 + G5 - see Figure III-B in the Data Supplement, containing discriminatory KOs) associated with Lachnospiraceae among LPD clusters. I) Heatmaps displaying significant (False Discovery Rate corrected, FDR $\leq$ 0.05) Spearman's rank correlations between MAGs and HDL subfractions. 
bioRxiv preprint doi: https://doi.org/10.1101/2021.09.01.458531; this version posted September 2, 2021. The copyright holder for this

preprint (which was not certified by peer review) is the author/funder, who has granted bioRxiv a license to display the preprint in perpetuity. It is made available under aCC-BY-NC-ND 4.0 International license.

Castro-Mejía et al.,

696
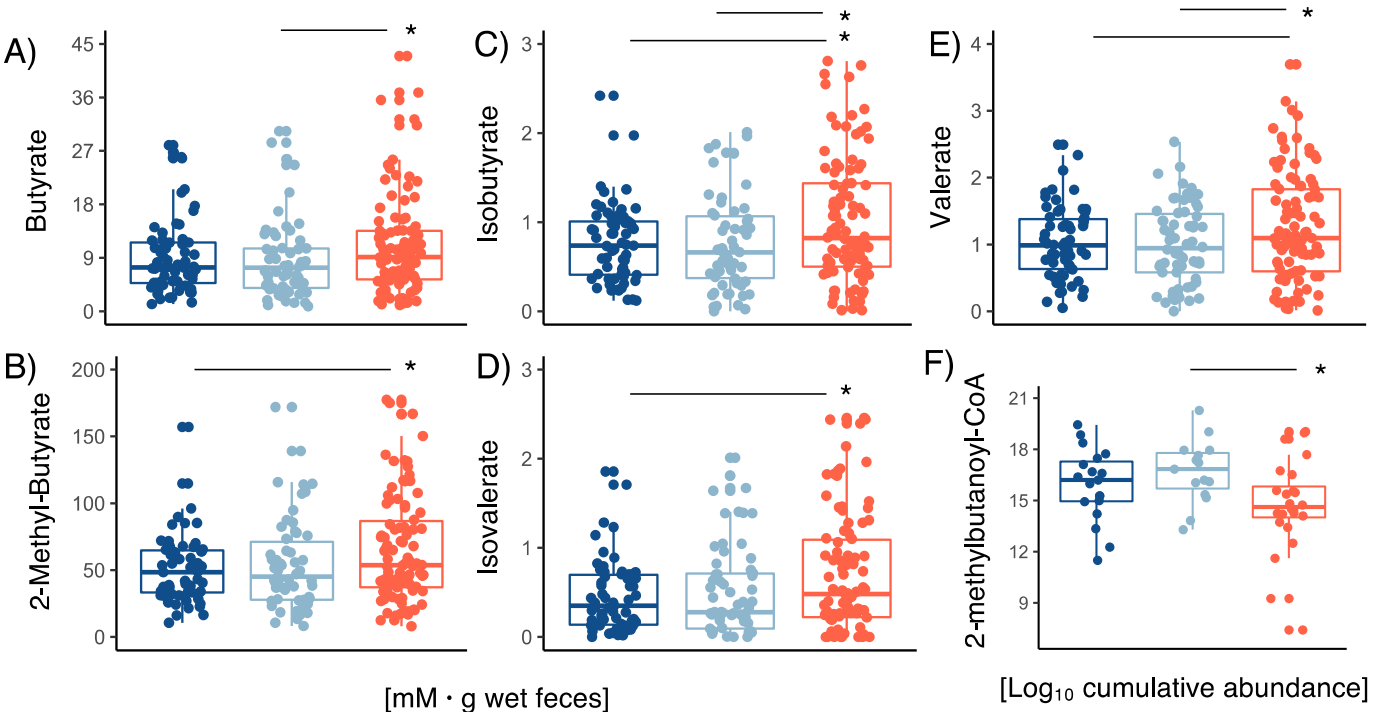

Figure 6. Short chain fatty acid concentrations

Range of fecal A) butyrate, B) 2-methylbutyrate, C) isobutyrate, D) isovalerate, E) valerate concentrations within the different LPD clusters. Cumulative abundance 2-methylbutanoylCoA genes screened on metagenomes within LPD clusters. Stars show statistical level of significance $(* p \leq 0.05)$ 